\title{
The Application Advantages of Microwave Fixation in Cotton Fabric Dyeing
}

\author{
Huang De-chao \\ (Dongguan Polytechnic, Guangdong, Dongguan, 523808)
}

Keywords: Microwave fixation; Tie-dye. Cotton fabric

\begin{abstract}
Gradually along with the application of microwave technology, microwave components performance improves, and the cost drops sharply, so the technology, with the attitude of high and new technology, is widely used in dyeing industry. This paper from raw materials and equipment of microwave dying, introduced microwave dyeing process and the influence on cotton fabric dyeing.

Tie-dye products are popular with most people because of its rich and colorful design and color, natural and unique shape of flower and breed design and color. This dye printing method can achieve tie-dye effect that other traditional tie-dyed techniques cannot achieve. Traditional techniques are only applicable in small batch and many varieties scale of production, and often with different fabric implementation means, such as sew, bundle, which leads to the result that dye solution cannot be evenly applied to different parts of the plant. Microwave dyeing is microwave heating technology. In addition to being applied to textile material drying operation,microwave technology also can be applied to dyeing and fixation treatment of printing and dyeing. Microwave for cotton fabric dyeing processing, dip the fabric in dye solution first, then put it in closed microwave regenerative chamber, based on the role of microwave technology on cotton dyed fabric, the dyes and auxiliaries molecules polarize or change again and again, after repeated friction heat between molecules, so as to achieve the purpose of rapid dyeing and color. Microwave dyeing technology heating is fast, and there is no heat loss and pollution of fabric. Microwave can guarantee the cotton goods inside and outside heat in a very short time with fabric dyeing uniformity, and good color fastness. Because microwave dyeing technology possesses the advantages of high efficiency and energy saving, and is easy to control, so, it is convenient for continuous production in textile industry.
\end{abstract}

\section{Microwave dyeing using raw materials and equipment}

This study chooses cotton bleaching fabric, uses anhydrous sodium sulfate (analysis of purity, Yao Hua Chemical Reagent Liability Co. LTD.in Tianjin) and anhydrous sodium sulfate (analysis of purity, Tanggu Chemical Reagent Factory in Tianjin), and the instruments and equipment are: in the production of Wenzhou Fangyuan Instrument Co., LTD: MU307A type horizontal experimental rolling cars, sw - 12A wash fastness tester, Y571D type multifunctional color fastness to rubbing;Galanz Electrical Appliances Industrial Co., LTD production: P70D2OTP - C6 type microwave oven, and so on.

\section{Conventional and microwave dyeing process}

A.Conventional dyeing process

1. The prescription of conventional dyeing process:

Active orange K - R \% (owf) 1

$\mathrm{Na} 2 \mathrm{SO} 4$ / (g/L) 50

$\mathrm{Na} 2 \mathrm{CO} 3 /(\mathrm{g} / \mathrm{L}) 10$

Bath rate1:30

2. Conventional process

The conventional dyeing process is shown in Figure 1. 


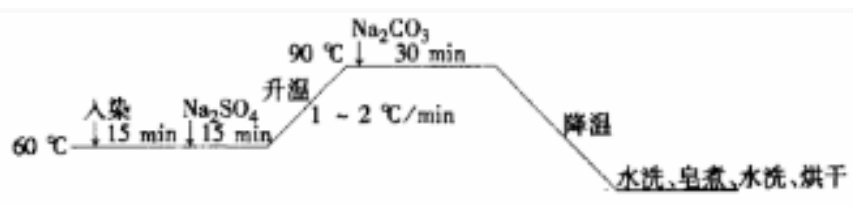

B.The condition of microwave dyeing

1. microwave dyeing solid color process

pad dyeing liquid $\rightarrow$ Preliminary drying processing (90 oC $* 1.5 \mathrm{~min}$ ) $\rightarrow$ microwave heating solid color $\rightarrow 80$ oC washing operation $\rightarrow$ soaping operation (4 g/L, 1:30, 95 oC * 3 min)

$\rightarrow$ washing $(80$ oC) $\rightarrow$ after cold water washing again, drying.

2.Dip-dye operation

Dye tested cotton fabric 15 min at 60 oc, add 1/2 salt, temperature up to 90 oc, fixation operation (at the same time adding soda ash, fixation time is $30 \mathrm{~min}$ ), specific washing operations are as 1.

3.Pad dyeing steps

Pad dyeing rolling rate is $80 \% 1 \rightarrow$ preliminary drying operation (90 oc * $90 \mathrm{~s}$ ) $\rightarrow$ operating environment set to 150 oc $* 2$ min $\rightarrow$ washing operations is as 1 .

\section{Cotton fabric dyeing test method}

A.Test microwave dyeing rate

In microwave cotton fabric dyeing solid color processing and conventional dyeing, sampleat intervals of $1 \mathrm{~min}$, dilute the sampling dyeing color page to $200 \mathrm{ml}$ by using distilled water, and test its absorbance. Dyeing percentage calculation formula is:

$E / \%=\left[1-\left(\frac{A_{n}}{A_{0}}\right)\right] * 100$

B. Strict testing cotton friction fastness

With the aid of Textile Color Fastness Rubbing Fastness Test GB/T3921-2008 test, use grey samplecolor cards for fastness and paste lining fabric color fastness assessment.

C.Test cotton fabrics soaping fastness

According to Textile Testing Fastness Soaping Fastness to GB/Y3921-2008 measurement, use grey cards for generalization.

D.Testing of K/S value and color features

With the help of computer color measurement instrument, test K/S value and color L *, a, b, c characteristics. Test environment lays 4 fold of the dyed fabric, the actual scan range control is in $400 \sim 700 \mathrm{~nm}$, and read the information of maximum absorption wavelength.

E.Test levelness of cotton fabric

Using computer color measurement instrument, according to the specification on the uniform color sampling principle of dyeing samples for processing, randomly test20 points brightness, purity, and $\mathrm{S}$ average deviation of tonal $\mathrm{H}$, the difference $\mathrm{P}$ in value between the maximum and the minimum in the different parts, thoroughly solve color difference on average, and judge the levelness of dyed cotton fabric according to the value of color difference.

\section{Microwave fixation effect of cotton fabric dyeing}

A.The influence of microwave radiation power on cotton fabric dyeing

Using the above formula, dye process for cotton fabric dyeing, microwave fixation time control is $8 \mathrm{~min}$, change the power of microwave radiation (300, 400, 500, 400, $500 \mathrm{w}$ ). Wecan see clearly the impact of the different microwave radiation power items color quantity, and the actual situation is shown in Figure 2. Using microwave technology for cotton fabric solid color processing, with the increase of microwave radiation power, the amount of color cotton rose, and when microwave radiation power is controlled in $700 \mathrm{w}$, the effect is the best. Cotton fabric microwave fixation 
treatment mainly uses the help of thermal effect mechanism, cotton fabric dye solution by microwave irradiation, appears repeatedlypolarization or changes gradually. Molecular fevers due to repeated friction, and at this point the microwave energy is converted into heat energy in the fast time. At this point, part of dye molecules with the effect of microwave easily appear induced and temperature rise. Dye molecules can quickly get the maximum kinetic energy and fiber expansion is gradually obvious, thus achieve the result of rapid dyeing and solid color.

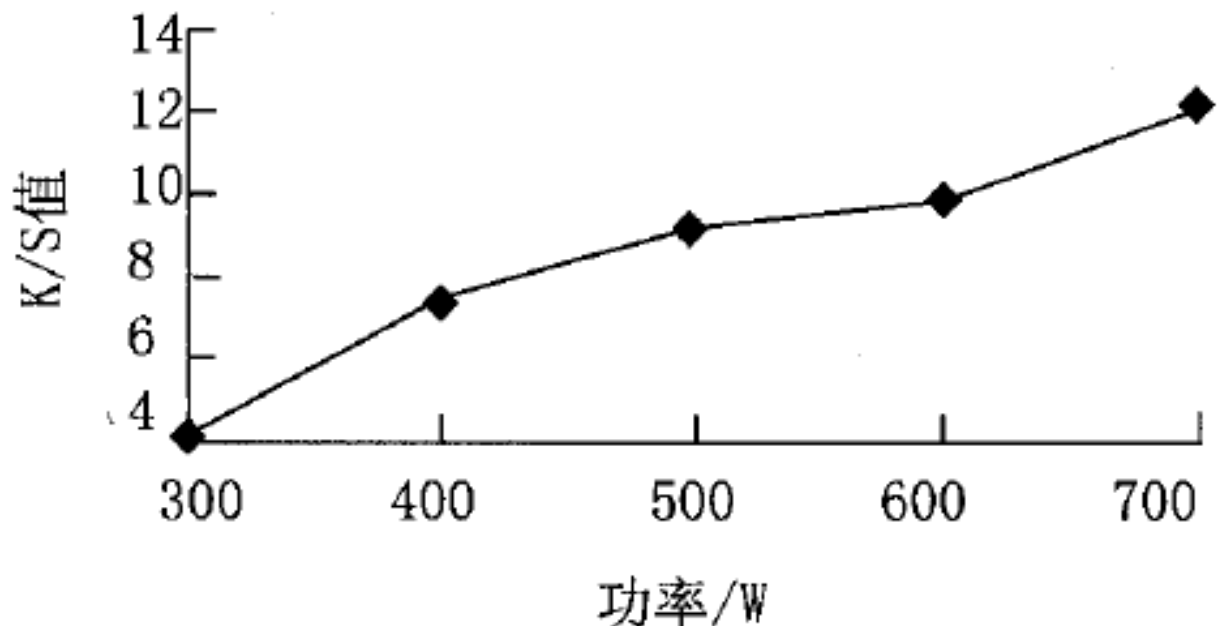

Figure 2 Microwave radiation power on dyed cotton K/S value

B.Microwave fixation time affects the quantity of color cotton goods

Use the dyeing recipe and process for cotton fabric dyeing operation, microwave power control is $700 \mathrm{w}$, change microwave fixation time, respectively, explore different microwave fixation time impact on cotton goods. We can see from Figure 3 that due to the increasing of microwave fixation time, the amount of cotton items color also gradually deepens. If time control is $8 \mathrm{~min}$, cotton goods whole microwave fixation has been basically completed, once again increase microwave fixation time, cotton fabric has no obvious color changes, microwave radiation processing can heat up in a very short time, and then achieve the effect of rapid dyeing cotton fabrics and solid color. Therefore, when microwave fixation time is $8 \mathrm{~min}$, the overall effect of cotton fabric is the best.

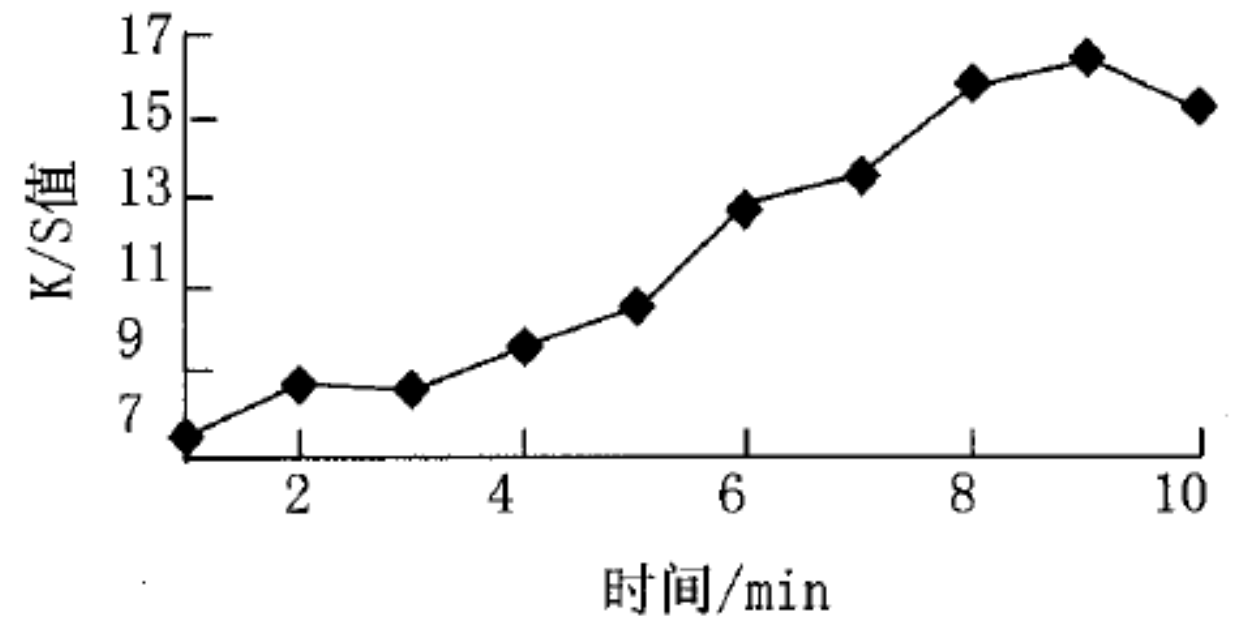

Figure 3 Microwave fixation time influence on cotton fabric color amount K/S value

C.Electrolyte dosage influence on cotton fabric color amount

While processing cotton fabric solid color with conventional reactive dyes, electrolyte has extremely important influence on dyeing effect. Using the above specification of dyeing process, electrolyte dosage in dyeing recipe is in the form of variable, respectively set the variables to 0,5 , 
15, 25, 35, $45 \mathrm{~g} / \mathrm{L}$, and according to the dyeing process,make color fixation operation for cotton fabric, and the particular case is shown in Figure 4. In the operation, the dosage of sodium sulphate increases from $0 \mathrm{~g} / \mathrm{L}$ to $35 \mathrm{~g} / \mathrm{L}$, cotton fabric color quantity increases, when the color amount rose to $45 \mathrm{~g} / \mathrm{L}$, cotton color amount declines. Accordingly, when dosage of sodium sulphate is $35 \mathrm{~g} / \mathrm{L}$, color quantity is high, which means that cotton dyed fabric color effect is the best.

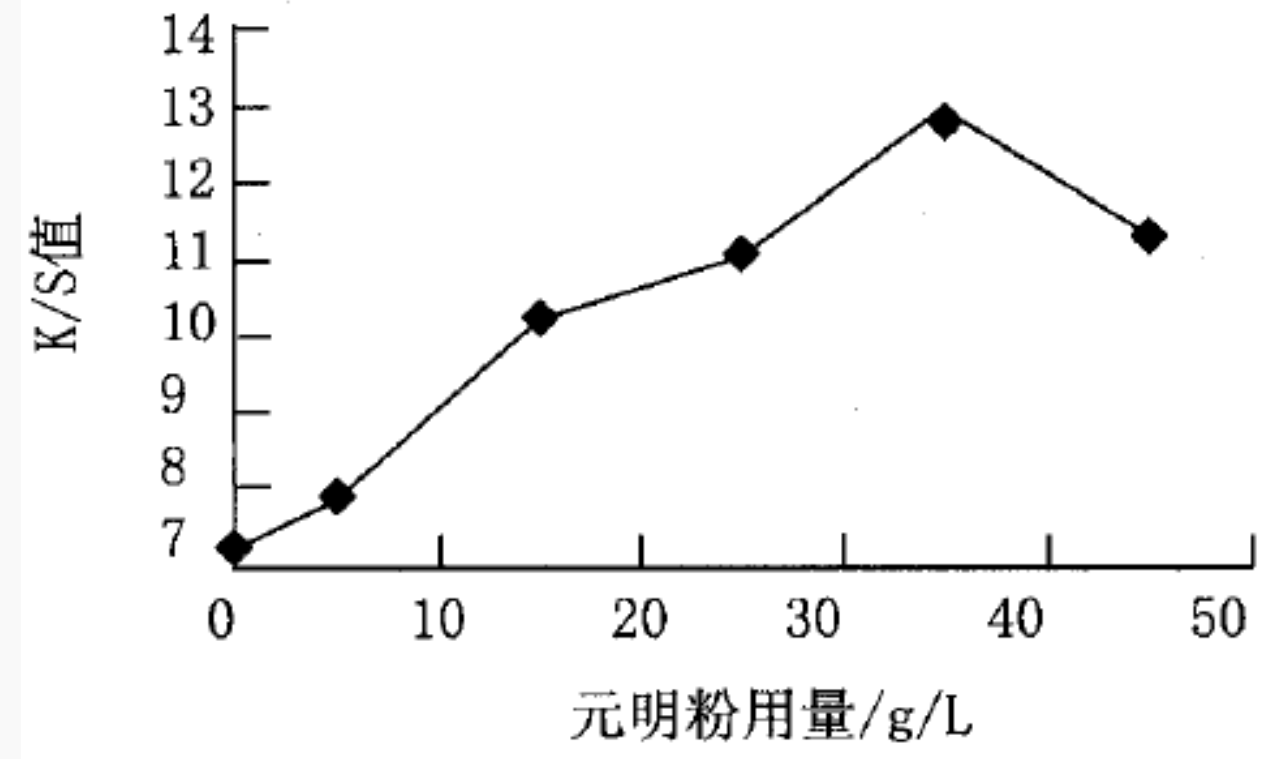

Figure 4 Electrolyte dosage influence on the amount of color cotton fabric

\section{The analysis of the advantages of microwave solid color used for cotton fabric dyeing}

A.Compare the effect of microwave and conventional piled solid color

Using the dyeing process for dyeing, microwave power and fixation time are set to $700 \mathrm{w}, 8 \mathrm{~min}$ respectively, as inTable 1.

Table 1The color amount of cotton fabric after dyeing

cotton fabric color quantity $\mathrm{K} / \mathrm{S}$ value after dyeing process

\begin{tabular}{lll}
\multicolumn{3}{l}{ Microwave solid colorpiled solid color } \\
\hline Active red HE-3B & 12.142 & 13.810
\end{tabular}

Table 1 shows that cotton fabrics dyed solid color should be carried out under the environment of operation. Because of the diffusion rate of dye itself is not high, solid color process is slow; Using microwave technology,do dyeing solid color processingon cotton fabrics, color fixation operations must be under the environment of high temperature, because microwave radiation is relatively uniform, and microwave solid colorneedshorter time. Under the environment of the normal temperature,do solid color processing. The presence of water molecules forms are layer or beam, microwave radiation causes great vibration within water molecules. By means of microwave technique,do fixation treatment on cotton fabric, set microwave fixation time to $8 \mathrm{~min}$, and it is closer to cotton pad dyeing piled up $24 \mathrm{~h}$ color quantity, so microwave technology fixation occupies unique advantage at this time.

B.The difference comparison of microwave heating solid color and conventional solid color

According to the standard process of dyeing process, do cotton fabric dyeing processing.Set microwave power to $700 \mathrm{w}$, and fixation time $8 \mathrm{~min}$.Compare effect of microwave heating solid color and conventional dyeing color amount, specific circumstances is in Table 2 . The table shows that conventional impregnation operating get the highest amount of color, microwave fixation the 
second, and the difference is not obvious, and conventional pad dyeing effect is the worst.

Table 2 Cotton fabric color amount after dyeing

cotton fabric color quantity $\mathrm{K} / \mathrm{S}$ value after dyeing process

Active red(1) microwave solid color(2) conventional impregnation(3) conventional dyeing

$\begin{array}{llll}\text { HE-3B } & 13.851 & 15.564 & 12.902\end{array}$

\section{C.Analysis of microwave levelness after fixation}

Table 3 shows that using microwave fixation treatment of cotton fabric, chroma tends to stable state, but its brightness $\mathrm{L}$ and color $\mathrm{H}$ exist corresponding fluctuation, which cause the overall average deviation S, average color difference, values are bigger. Therefore no conventional dyeing levelness effect is good.The above situation is due to not high homogeneous degree of rolled car rolled out, and poor levelness caused by degree of microwave.

Table 3 Analysis of cotton fabrics levelness after microwave fixation

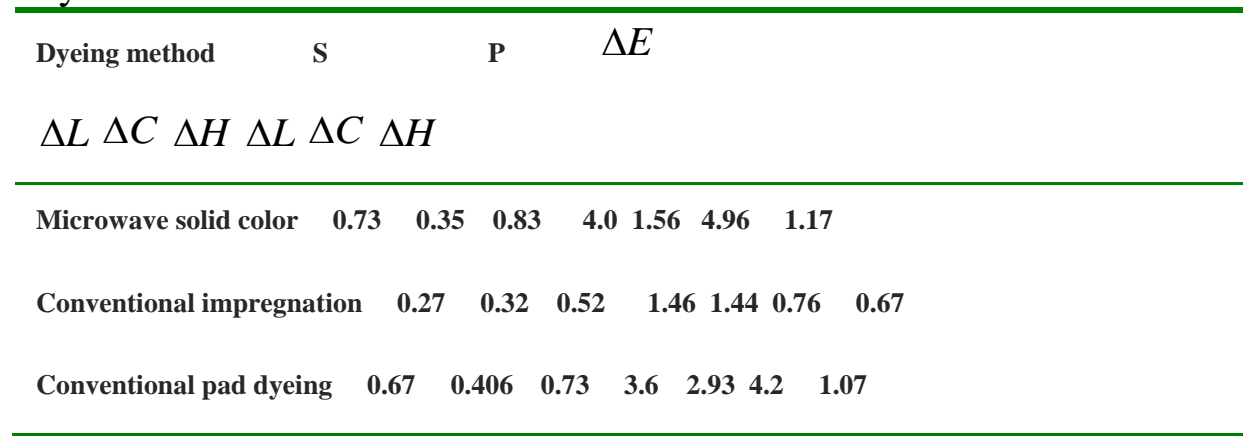

\section{Conclusion}

Above all, do cotton fabric solid color by microwave heating technology.As the change of microwave irradiation power and time, the color amount of cotton fabric will also change. After microwave solid color, cotton fabric has good levelness and color fastness.Microwave dyeing solid color processing has good feasibility, low energy consumption, green environmental protection and other characteristics, which can be promoted to the field of industrial production.

\section{References}

[1]Su Yi. Cotton pad dyeing microwave fixation technology research [J]. Journal of Dyes and Dyeing, 2012,49 (2) : 23-26.

[2] Yu Zhong. Silk wool textiles tie-dye technology and art study [J]. Journal of Wool Spinning Technology, 2011, 33 (8) 6:17-18.

[3] Luo Weixin, Wang Lugang, ChuYunRong etc. Wool/cotton/viscose blended fabric pretreatment process [J]. Journal of Printing and Dyeing, 2013, 33 (4) 6:21-23.

[4] Song Xinyuan. Charge effect and cationic reagents development and application of textile dyeing (to continue) [J]. Journal of Printing and Dyeing Auxiliaries, 2012, 29 (5) : 1-9.

[5] Na Bin, Wang Zhipeng, Sun Dandan etc. Two methods of fast-growing poplar veneer dyeing processing [J]. Journal of Northeast Forestry University, 2011, 33 (6) 6:41-43.

[6] Liu Yuanjun,Wang Xueyan, Song Bingzheng etc. WLS and WLS-20 modified cotton fabric dyeing effect evaluation [J]. Journal of Xi 'an Engineering University, 2012, 26 (5) : 576-580.

[7] Hong Hua, Shen Xiaoping, Li Peicai etc. Dyeing and finishing process effects residues of pesticide on cotton textile research [J]. Chemical Analysis Measurement, 2014, (1) : 27 to 30. 